\title{
Ethical Issues Associated with the Use of Animal Experimentation in Behavioral Neuroscience Research
}

\author{
Frauke Ohl and Franck Meijboom
}

\begin{abstract}
This chapter briefly explores whether there are distinct characteristics in the field of Behavioral Neuroscience that demand specific ethical reflection. We argue that although the ethical issues in animal-based Behavioral Neuroscience are not necessarily distinct from those in other research disciplines using animal experimentation, this field of endeavor makes a number of specific, ethically relevant, questions more explicit and, as a result, may expose to discussion a series of ethical issues that have relevance beyond this field of science. We suggest that innovative research, by its very definition, demands out-of-the-box thinking. At the same time, standardization of animal models and test procedures for the sake of comparability across experiments inhibits the potential and willingness to leave well-established tracks of thinking, and leaves us wondering how open minded research is and whether it is the researcher's established perspective that drives the research rather than the research that drives the researcher's perspective. The chapter finishes by introducing subsequent chapters of this book volume on Ethical Issues in Behavioral Neuroscience.
\end{abstract}

Keywords Animal behavior - Translational value $\cdot$ Animal ethics · Animal model

\section{Contents}

1 Reasons for Reflection?

2 The Moral Status of Animals as a Start of Ethical Concerns About Their Use in Experiments

3 Relevance of Animal Models? .............................................................................

4 Thinking Out-of-the-Box ....................................................................................... 11

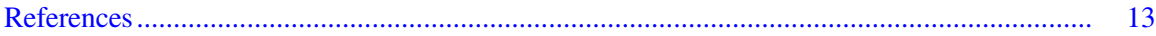

F. Ohl $(\bowtie) \cdot$ F. Meijboom

Department Animals in Science \& Society, Faculty of Veterinary Medicine,

University Utrecht, Yalelaan 2, PO Box 80.166, 3508 TD Utrecht, The Netherlands

e-mail: f.ohl@uu.nl

F. Meijboom

Ethics Institute, Utrecht University, Utrecht, The Netherlands

Curr Topics Behav Neurosci (2015) 19: 3-15 


\section{Reasons for Reflection?}

The first part of this book on Ethics in Behavioral Neuroscience explores the question of whether it is worthwhile, or even necessary, to reflect specifically on animal experimentation in Behavioral Neurosciences in extension of more general considerations on Animal Ethics in the broader sense. Are there distinct characteristics in this field of research that demand specific ethical reflection?

Of course, there is an obligation to reflect on the use of animals as models in Behavioral Neuroscience. But, research on animals has already triggered considerable attention during the last decades, exploring whether it may be justifiable to use animals for experiments at all and, if so, how to weigh the costs of such use against its benefits (e.g. Singer 1975; Van Zutphen et al. 1993; Brom 2002; Nuffield 2005) and these same questions hold for other areas of research and are not unique to the field of Behavioral Neuroscience.

More recently however, Neuroethics has emerged as a distinct field of applied ethics within the philosophy of neuroscience (Stefansson 2007; Illes and Sahakian 2011). Neuroethics deals with a wide range of questions related both to the ethical implications of practical experimentation in neuroscience and the application of the results of such neuroscientific research as well as, in turn, the consequences of neuroscience for ethics (cf. Roskies 2002; Buller 2014). In practice however, it appears that, to date, these discussions have mainly focused on humans - as for example, discussions on the moral rights and wrongs of the enhancement of brain function, or questions related to the concept of free will and moral agency. Thus, although Behavioral Neuroscience does raise specific ethical questions in relation to experimental animal research, the attention of neuroethicists has not, at least to this point, been specifically concerned with this wider context of the ethics of animal experimentation in neuroscience.

Yet there are very specific issues which are raised by the use of animal experiments in this particular area of neuroscience; it is because of those specific aspects, which lie in the interactions between the fields of animal ethics and neuroethics, that we consider it relevant to dedicate a section of the book to the ethical issues of animal-based research in Behavioral Neuroscience. Alongside the more basic questions of animal ethics, a research field that is often dependent on modeling distinct mental capacities and behavioral responses in animals, may have specific implications on considerations on the moral status of animals. Thus, the very criteria that lead us to judge some animal a valid research model in Behavioral Neuroscience are pretty much the same as we would use to grant animals moral consideration for their own sake, which inevitably leads to some conflict in terms of the acceptability of their use for experiments.

Therefore, we argue that although the ethical issues in animal-based Behavioral Neuroscience are not necessarily distinct from those in other research disciplines using animal experimentation, this field of endeavor makes a number of specific, ethically relevant, questions more explicit and, as a result, may expose to discussion a series of ethical issues that have relevance beyond this field of science. 
In addition to the conflict which may result from the fact that the most valid animal models may also be those which we might consider, from those same characteristics, as having the highest claim to be worthy of specific moral consideration, other questions may, for example, be related to the predictive power of specific animal models and the degree to which results gained on those models may be truly translated to other systems or species (including humans) (Rollin and Rollin 2014). How should we deal with uncertainties regarding the predictive and construct validity of given (animal) models (cf. Geyer and Markou 1995)? How much research is needed before it is justified to move from work on animals to take the step into (pre)clinical trials? And finally: how can we balance the potential benefit of using animal models that might have higher mental capacities (thus enhancing possible translational value to humans) against the cost that such higher mental capacities may imply greater suffering as the result of experimental manipulations?

This chapter briefly introduces ethical questions raising from animal-based Behavioral Neuroscience, each of which will be developed in more detail in the subsequent chapters of this section.

\section{The Moral Status of Animals as a Start of Ethical Concerns About Their Use in Experiments}

The use of animals in experimental research in general has raised many concerns over the years. While perhaps earliest concerns about experimentation involving live animals arose in the UK in the nineteenth century (Franco 2013), debate about the moral status of animals is not restricted to Europe, but is nowadays of concern in many countries including the US, Australia and Asian countries (cf. Bovenkerk 2012; Linzey 2014; Nuffield Council 2005). The origin of these discussions lies in the recognition of animals as moral subjects toward which we can have moral duties (Warren 1997). A significant number of ethicists concede that animals have some moral value that is independent of their use by humans. However, there is a diversity of arguments that underlie the recognition of this moral standing of animals. Some start in the recognition of animals as living beings that have a good of their own. This is based on the idea that animals develop, maintain their life, and can adapt successfully to their environment. As a consequence, they have inherent worth as animals (Taylor 1986). Others argue for the moral considerability of animals by virtue of their being able to feel (e.g. Rollin 2011)

It is beyond the scope of this chapter fully to elaborate on the diversity of views that have characterized the debate in the past few decades (Callicott 1980; Carruthers 1992; DeGrazia 1996; Midgley 1983; Korsgaard 2005; Nussbaum 2006; Regan 2004; Rollin 1981; Rowlands 2002; Singer 1995) —and these arguments are rehearsed in greater detail in later by Bovenkerk and Kaldewaij (this volume) and Vieira de Castro and Olsson (this volume). However, both within the field of animal 
ethics and in formal regulations on the use of animals in research there is a consensus that we have valid and sufficient reasons to consider animals as legitimate objects of our moral concern (cf. De Cock Buning et al. 2009 ; EU 2010).

In a nutshell, such recognition implies that animals should be taken into account in our moral reasoning for their own sake. In animal research the health and welfare of animals is of course taken into account, because compromise of either state may frustrate the research or influence the results in some way. However, speaking about animals as moral subjects implies a further step: if animals are acknowledged to be worthy of consideration and significant entities in their own right, we have direct moral reasons to ensure that our actions take account of their interests as well as our own. How this consideration can be translated into practice is not always immediately clear. Some argue that, as a consequence, any type of animal research is unacceptable (Regan 2004). Others stress that there are also legitimate ethical positions that aim to take the interests or value of animals seriously, yet do not exclude the option that using animals for research can morally be justified (cf. Rollin and Kessel 1990; and see Rollin, this volume; Vieira de Castro and Olsson, this volume). This implies that, on the one hand, using animals is not something that is to be rejected by principle; on the other hand, although animals continue to be used, such use demands a careful consideration.

Frequently, such consideration is based on an analysis of the comparative costs (i.e., harm to individual animals) and benefits (see again Vieira de Castro and Olsson, this volume). Determining the moral justification of animal research in terms of such cost-benefit analysis, in effect gives particular emphasis to two central questions: does the expected result of the experiment or project outweigh the potential suffering of the animals; and is the experiment being performed in the best possible way with regard to the principles of Replacement, Reduction and Refinement (Russel and Burch 1959). Such an evaluation process implies that the ethical justification of animal experiments demands that there shall be specific benefits as a result of any experiment that are considered important enough to outweigh the costs for the animal. In general, the benefit of using animals in experiments is argued in terms of its contribution towards reduction of suffering in humans as an immediate or ultimate aim. This holds equally for experimental animal research in Behavioral Neuroscience.

The majority of such experiments is aimed, if sometimes indirectly, at gaining knowledge about the executive function of the brain. Most commonly, it is the dysfunctioning of particular processes that is of especial interest, because some specific dysfunction of the CNS underlies a variety of disorders that can have a severe impact on (human) quality of life. Since many ethical frameworks stress that we have a duty to take action in the face of human suffering, there is a moral imperative to perform some form of research in this field. Having accepted such duty to care for the health and wellbeing of humans, however, there is no automatic logical presumption that animals have to be used or that use of animals is automatically justified. Therefore, an important aspect of the ethical justification of animal experimentation is discussion both of the need to use animals at all and on the relevance of animal models in research (to ensure that animals used genuinely 
do provide appropriate models for human systems or disorders, rather than simply mimicking symptoms but in an unrelated way). We should, therefore, take a closer look at the validity of the animal models used in this field of research, and their relevance for transference of results to other systems and species.

\section{Relevance of Animal Models?}

The actual relevance of animal models for a distinct field of research is difficult to assess. One may get some impression of the current [quantitative] importance of animal models in experimental Behavioral Neuroscience by way of a literature research, although, of course, there is virtually no way to assess whether the use of particular animal models employed, has indeed resulted in relevant output. Given such reservations, however, it appears from a rough and explorative online screening for recent literature, that of the 7,500 original research articles that have been published on this topic during the last 5 years (PubMed 2009-2013), more than $40 \%$ of the papers at least make some reference to animal models. More specifically, PubMed reports the following number of articles published in the last 5 years when searching with the key-phrase "behavioral neuroscience" together with $[\ldots]$ :

\section{[humans]: 2400}

[either humans or other animals and (computer modeling)]: 56

[either humans or other animals and (in vitro)]: 190

[other animals]: 3665

While such numbers cannot tell us anything about the actual contribution of animal studies to developments, and valid advances, within this field of research, such an overview suggests that studies in humans and animals each contribute almost equally to the overall publication output in neurobehavioral research. Given all the recent technical developments and the range of opportunities now available to perform non-invasive experiments in humans, as well as to model neural processes in vitro, it seems somewhat intriguing that animal-based experiments continue to play such a big role in Behavioral Neuroscience. For this to remain true, the results gained from animal experiments in Behavioral Neuroscience are obviously assessed, at least by the researchers themselves, or the wider research community, as of importance - perhaps because they are thought to contribute as much to the development of the research field as do studies in humans, or perhaps for other reasons. It may, for example, be that animal experiments are considered more ethically acceptable than pre-clinical studies in humans; it is also possible that research, or at least the publication of research, constrains itself by following distinct traditions, such as demanding the validation of novel findings by comparing them to already published animal models and test procedures.

One significant question arising from the continued extensive use of animals is embedded in the broader debate on the possibility of replacement of animal 
experiments, the first of the 3R-principles (Russel and Burch 1959). Although, the search for animal-free methods is complex in any research field (Doktorova et al. 2012; Hendriksen 2009; Huggins 2003; Manciocco et al. 2009; Penza et al. 2009), in Behavioral Neuroscience in particular, the modeling of complex systems such as executive processes of the brain or the central nervous system (CNS) may indeed limit the possibility of finding alternatives and may thus demand use of animal models; at least at present, available in vitro methods, and computer models seem unable to display the complexity of CNS-generated, behavioral-cognitive processes. It may be of note, however, that the declared goal of one of the current EU flagship programs (the Human Brain Project) is: "to build a completely new ICT infrastructure for neuroscience, and for brain-related research in medicine and computing, catalyzing a global collaborative effort to understand the human brain and its diseases and ultimately to emulate its computational capabilities."1

While waiting for the results of such initiatives, the use of methods that avoid the use of live animals is still quite limited. But even if we do accept the need to base parts of research in Behavioral Neuroscience on the use of animals, some ethically relevant questions remain to be considered. And first among these questions, as above, is: what it is that animals are supposed to model and are we choosing the correct models?

If we look in more detail at the specific areas of animal experimentation, a literature search using the term "animal model" in combination with some general topics reveals that use of animal models in many cases is related to research into a variety of human-specific, mental disorders. Such a literature scan, again performed on articles listed by PubMed and over the same time period, picks out the following number of publications with the combined keywords [animal model] and [...]:

[stress]: 13561

[alzheimer]: 2568

[depression]: 2918

[schizophrenia]: 1464

[anxiety]: 2340

[mood disorder]: 982

[hyperactivity]: 924

[addiction]: 868

[post traumatic stress disorder]: 247

[eating disorder]: 219

This simple screening results in the identification of more than 25,000 articles on this (artificial) selection of human mental states/disorders. [For comparison: a search on [animal model] and [cancer] delivers 20,304 hits]. Without going too far in interpreting such a crude literature search, we may feel confident enough to suggest that animal models are still considered important in investigating human mental states and/or functions; indeed this use of animal models in exploration of

1 see https://www.humanbrainproject.eu/. 
human mental function comprises the majority of those animal studies uncovered in our initial literature review.

From any consideration of the ethics of animal experimentation, such extensive usage of animals begs the question as to whether the obvious importance of animal models genuinely translates into actual useful and relevant output, since the assumption that animals are relevant models can be seen as a pivotal argument in the moral justification of animal use (Rollin and Rollin 2014). A realistic assessment of the benefits and, thus, actual relevance of animal studies is however, more or less impossible to do in practical terms (as explored in more detail in this volume by Viera de Castro and Olsson). Yet the very assumption that the animals chosen as models are valid and, thus, relevant models for human mental problems (such as distinct cognitive and emotional capacities) may indicate that these animals share with us morally relevant characteristics that may make them (more) worthwhile protecting, promoting additional concerns about their use in experimental treatments.

Given the need in Behavioral Neuroscience to model complex systems, and perhaps even integrate executive processes, such as learning and social behavior, it may be argued that the best choice for an animal model is the use of animals with 'higher' cognitive capacities, such as primates or dogs. However, as we have noted already, the scientific argument that these animals serve as relevant models because of the greater physiological or behavioral similarity to humans, as compared to other species like fruitflies or mice for example, is often the basis of public concerns because of exactly these same characteristics. As a result, experiments on primates and dogs often raise stronger societal resistance than experiments on rodents or fish (Hagen et al. 2012). In practice this complicates the discussion on the choice for the best possible animal model for a distinct experiment, as in fact the choice of the 'best possible' animal model becomes an interplay between value and scientific judgments.

In this context, it might be of interest to get some idea on what animal species actually are being used to investigate human mental disorders. Once again, we have used PubMed to search for all articles in PubMed which use again [animal model], but this time with [anxiety]. This search delivers 2,340 hits for publications between 2009 and 2013; repeating the same search with reference to individual species gives the following numbers of publications:

[mice or mouse]: 998 (355 on [C57BL])

[rat]: 970 (369 on [Wistar])

[primate]: 615

[fish]: 59

[dog]: 9

[rabbit]: 2

Although surely not fully representative, these findings are at least indicative of current patterns of research publication based on experiments using different animal species: first, we may note that about $25 \%$ of publications within this specific area of research refer explicitly to primates. This high proportion undoubtedly overpresents the number of experiments actually done in primates, since the proportional 
representation in publications reported here does not reflect the distribution of species reported as being used in research (reported for example by the EU in 2010 as: mice $59.3 \%$; rats $17.6 \%$; other rodents including guinea pigs and rabbits $5.2 \%$; ungulates $1.4 \%$; cats, dogs and other carnivores $0.3 \%$; and non-human primates $0.08 \%$; birds, reptiles, amphibians, and fish taken together $15.9 \%$; see Hagen et al. 2012). Secondly, and perhaps not surprisingly, experiments on mice and rats dominate the report on actual animal use (rats and mice combined, around $70 \%$ ) and, in this case are represented to about the same extent of (again combined) roughly $60 \%$ of published articles.

What is interesting though is that more than a third of publications on mice refer specifically to the inbred strain C57BL, and that about the same proportion of rat studies seem to involve the Wistar strain. Further, when we look at methodologies employed in experiments, our literature screening on [animal model] with [anxiety] and now specifying [elevated plus maze, or open field, or dark light box] results in 769 hits (again about one-third of the total of 2,340 hits). Overall, there seems to be at least some indication that animal experiments in anxiety research, as merely one example, is being based to a significant extent on only a small number of test systems and primarily on experiments on one distinct mouse or rat strain, respectively.

Such considerations may be of special relevance when considering future developments in experimental Behavioral Neuroscience research. It is predicted that mood disorders in humans, as for example clinical depression, will become one of the leading causes of disability worldwide (Murray and Lopes 1997; Rodríguez et al. 2012). Such a prediction increases the drive to understand better the development and underlying mechanisms of such disorders in order to develop better prevention and treatment; this, in turn, may increase the requirement or motivation to undertake more research, in all probability based in the same way on the use of animal models. This potential development focuses further a debate on the appropriateness and validity of models currently used.

While we would not want to overstate the implications from this limited survey -a more rigorous analysis would clearly demand a much more extensive literature research - we may at least wonder whether indeed the combination of these test systems and strains is genuinely believed to deliver the best possible results in anxiety research or is simply based on tradition, conservatism and lack of exploration of alternative models - or acceptability to journals and their equally conservative referees. To us it seems important at least to raise the question as to whether animal-based research may be self-perpetuating as the result of unimaginative and conventional thinking regarding the choice of animal models and test systems used, and whether such conventional choices are truly the best possible choices in the search for innovative research findings. Gold standards surely have their use, but we should not forget that such standards are established within the frames of knowledge at their time of establishment. Scientific knowledge however develops rapidly — or so we hope - and it may be reasonable to wonder about the half-life of any gold standard, before it turns into fool's gold. 
In a recent review article on the predictive value of animals models McGonigle and Ruggeri (2014) state: "For major mood disorders, such as depression and anxiety, inadequacies in the animal models have helped undermine the confidence of major pharmaceutical companies to the point that several, if not the majority have either withdrawn from this therapeutic area or significantly reduced their internal research activities." Indeed it seems of crucial importance not only to try and optimize procedures of animal-based research as such, but carefully to evaluate how appropriate is the model chosen and, in this way not only optimizing the translational value of studies in animal models, but also allowing for actual, retrospective assessment of such translational value. McGonigle and Ruggeri conclude from their review that "Comparison of models within a given therapeutic area, approaches to models and cross fertilization between therapeutic areas will do much to improve translational research. By thinking outside the box that each therapeutic area has created, improvements will be made to existing models to make these more predictive. These advances will inform both the development of new models and biomarkers that will enhance the translational relevance as well as the predictive utility of pre-clinical animal models of human disease, irrespective of therapeutic area."

\section{Thinking Out-of-the-Box}

Research claims to be innovative, with the exception of experiments that are being done to confirm previous findings. But innovative research, by very definition, demands out-of-the-boxthinking. At the same time standardization of animal models and test procedures for the sake of comparability across experiments inhibits the potential and willingness to leave well established tracks of thinking. Indeed, as Rob Hutter states: “...today's neuroscience research can be described as 'what happens' research versus 'how to make happen' research. One could argue that the former precedes the latter, but there are perspective issues that drive the type of questions researchers are likely to ask as well as the scope of tasks and behaviors that can be included in rigorous experimental conditions." $"$ We may thus wonder how open minded research is and whether it is the researcher's established perspective that drives the research rather than the research that drives the researcher's perspective.

In search of the best possible research results in animal-based Behavioral Neuroscience and, thus, in trying to optimize the benefit of animal experiments, while at the same time minimizing the costs, any innovative perspective will be closely linked to the choice of the animal model used. Is it, for example, necessary for an animal to being able to perceive pain in order to resemble a valid animal

2 DO.Anything; The Science of Intentional Change, posted by Rob Hutter, January 2013; http:// robhutter.com/neuroscience/the-neuroscience-of-behavioral-insight/. 
model for pain research? Bernard Rollin (this volume) suggests that "the modification of telos by way of combining genetic engineering with behavioral neuroscience as a remedy for practices that cause pain or suffering by violation of telos represents a whole new approach to intractable problems of animal welfare that emerge from contemporary animal use" and is supported in this by Adam Shriver (this volume) who argues "that we already have, or are extremely close to having, the capacity to dramatically reduce the amount of suffering caused in biomedical research via genetic modification of the animals used in research." The appropriate selection, or perhaps even creation of animal models thus deserves special attention in relation to options for reducing the potential for animal suffering, in relation to the improvement of animal welfare and the considerations of animal integrity (cf. Van der Staay et al. 2009). Such evaluation processes may, however, also profit from some out-of-the-box thinking and the subsequent chapters in this book are intended to stimulate such out-of-the-box thinking in animal-based Behavioral Neuroscience.

Bernice Bovenkerk and Frederike Kaldewaij make a start by reflecting on the tension between the need for translatability in animal models and the moral status of animals. They invite us critically to think about some justifications for the claim that human beings and more complex animals have superior moral status and argue that contemporary approaches which attribute equal moral status to all beings that are capable of conscious strivings (e.g., avoiding pain and anxiety; aiming to eat and play) are based on more plausible assumptions. They further suggest that, while there might be good reasons to assume that more complex beings would be harmed more by a specific physical or environmental intervention, it may also be possible that higher cognitive capacities result in less harm, because of a better ability to cope.

The ultimate use and validity of animal models would require to prove that indeed their use achieves its objective, that is that the results of a given animal study is a benefit that could not be gained otherwise. Ana Catarina Vieira de Castro and Anna Olsson in their chapter explore how cost-benefit analyses currently are being approached, and they conclude that specific 'costs' of animal experimentations in terms of harms inflicted on the animals, are far easier to assess that their benefits - a problem that actually may not be specific for Behavioral Neuroscience. Still, as outlined above, Behavioral Neuroscience often may affect the emotional and/or cognitive state in animals used, and such harm is difficult to counteract. Olsson and Vieira de Castro however come to the conclusion that effective cost-benefit analysis suffers from a lack of realistic ability to assess the true benefits and provocatively suggest that perhaps the benefit assessment should be discarded from any procedural ethical consideration, which, instead, should focus exclusively on the three Rs and improving animal welfare.

Paula Droege and Victoria Braithwaite continue with "a cross-disciplinary debate about the sort of framework that will best organize the growing body of data on behavior, development and anatomy of fish and other non-human animals in order to assess the capacity for consciousness." Fundamentally, considerations on how to assess consciousness in the first place remind us that a taxonomic classification of 'higher' and 'lower' species may be a poor guideline for the assessment 
of a species capacity to suffer. Instead, as Droege and Braithwaite state, only "once we have a means of determining what sorts of animals feel conscious pain, we can more effectively think about ways to minimize or eliminate their suffering."

Bernard Rollin then reflects on the question why we would consider it ethically problematic or even unacceptable to eliminate an animals' capacity to suffer by means of genetic manipulation, if we do find it acceptable to cause such suffering in the first place? "In biomedical research, we do indeed inflict major pain, suffering and disease on animals. And genetic engineering seems to augment our ability to create animals to model diseases, particularly the more than 3,000 known human genetic diseases. [...] Perhaps one can use the very genetic engineering which creates this dilemma to ablate consciousness in such animal models, thereby escaping a moral impasse." Underlying Rollin's considerations is the understanding that it is the individual one can wrong, not the telos.

In the concluding chapter, Adam Shriver explores how genetic manipulation of animals in order to reduce the animal's capacity to suffer would translate into experimental practice. What would be the benefit wnd what the costs of such manipulation? And would the elemination of the animal's capacity to suffer not be the most logical way to solve ethical dilemmas in experimental animal research?

As Bovenkerk and Kaldewaij state in their conclusions: "We have not attempted to give definitive answers here, but rather to raise some moral issues and to point out normative assumptions made in animal experimentation in general, and neurobehavioral research in particular." Indeed, ethical issues, as opposed to neurobehavioral questions, cannot be answered by way of statistical significance, but demand an ongoing and constructive discussion, to which we hope to contribute with this book.

Acknowledgments The authors wish to thank Rory Putman for helpful comments on the manuscript.

\section{References}

Bovenkerk B (2012) The biotechnology debate: democracy in the face of intractable disagreement, library of ethics and applied philosophy, vol 29. Springer, Dordrecht

Brom FWA (2002) Science and society: different bioethical approaches towards animal experimentation. ALTEX 19(2/02):78-82

Buller T (2014) Bridging the gap between science and ethics? cambridge quarterly of healthcare ethics, special section: neuroethics and animals animal minds and neuroimaging, vol 23. Cambridge University Press, pp 173-181. doi:10.1017/S0963180113000704

Callicott JB (1980) Animal liberation: a triangular affair. Environ Ethics 2-4:311-338

Carruthers P (1992) The animals issue: moral theory in practice. Cambridge UP, Cambridge

De Cock Buning Tj, Meijboom FLB, Swart JAA (2009) Ethiek en Dierproeven. In: van Zutphen LFM (ed) Handboek proefdierkunde. Proefdieren, dierproeven, alternatieven en ethiek. Elsevier, pp. 321-334

DeGrazia D (1996) Taking animals seriously. Mental life and moral status. Cambridge UP, Cambridge 
Doktorova TY, Pauwels M, Vinken M et al (2012) Opportunities for an alternative integrating testing strategy for carcinogen hazard assessment? Crit Rev Toxicol 42:91-106

EU (2010) Directive 201/63/EU on the protection of animals used for scientific purposes. Official Journal of the European Union L 276/33

Franco NH (2013) Animal experiments in biomedical research: a historical perspective. Animals 3:238-273

Geyer MA, Markou A (1995) Animal models of psychiatric disorders. In: Bloom FE, Kupfer DJ (eds) Psychopharmacology: the fourth generation of progress. Raven, New York, pp 787-798

Hagen K, Schnieke A, Thiele F (eds) (2012) Large animals as biomedical models: ethical, societal, legal and biological aspects. Europäische Akademie, Ahrweiler

Hendriksen CFM (2009) Replacement, reduction and refinement alternatives to animal use in vaccine potency measurement. Expert Rev Vaccines, 8:313-322

Huggins J (2003) Alternatives to animal testing: research, trends, validation, regulatory acceptance. Altex-Alternativen Zu Tierexperimenten 20(Supplement 1):3-61

Illes J, Sahakian BJ (eds) (2011) Oxford handbook of neuroethics. Oxford University Press, Oxford Library of Psychology, England

Korsgaard C (2005) Fellow creatures: Kantian ethics and our duties to animals. Tanner Lect Human Values 25:77-110

Linzey A (ed) (2014) The global guide to animal protection. University of Illinois Press, Illinois

Manciocco A, Chiarotti F, Vitale A et al (2009) The application of Russell and Burch 3R principle in rodent models of neurodegenerative disease: the case of Parkinson's disease. Neurosci Biobehav Rev 33(1):18-32

McGonigle P, Ruggeri B (2014) Animal models of human disease: challenges in enabling translation. Biochem Pharmacol 87(1):162-171. doi:10.1016/j.bcp.2013.08.006

Midgley M (1983) Animals and why they matter: a journey around the species barrier. University of Georgia Press, Athens

Murray CJ, Lopez AD (1997) Alternative projections of mortality and disability by cause 1990-2020: global burden of disease study. Lancet 349:1498-1504

Nuffield Council on Bioethics (2005) The ethics of research involving animals. Latimer Trend \& Company Ltd, London. (www.nuffieldbioethics.org)

Nussbaum MC (2006) Frontiers of justice. Disability, nationality, species membership. Harvard University Press, Cambridge

Penza M, Jeremic M, Montani C et al (2009) Alternatives to animal experimentation for hormonal compounds research. Genes Nutr 4:165-172

Regan T (2004) The case for animal rights, Updated with a new Preface, The University Press Group Ltd

Rodríguez MR, Nuevo R, Chatterji S, Ayuso-Mateos JL (2012) Definitions and factors associated with subthreshold depressive conditions: a systematic review. BMC Psychiatry 12:181

Rollin BE, Kessel ML (eds) (1990) The experimental animal in biomedical research. CRC Press/ Taylor \& Francis, Boca Raton

Rollin BE (1981) Animal rights \& human morality. Prometheus Books, New York

Rollin BE (2011) Animal pain: what it is and why it matters. J Ethics 15:425-437

Rollin MD, Rollin BE (2014) Crazy like a fox: validity and ethics of animal models of human psychiatric. Disease Cambridge quarterly of healthcare ethics, special section: neuroethics and animals animal minds and neuroimaging, vol 23. Cambridge University Press, pp 173-181. doi:10.1017/S0963180113000704

Rowlands M (2002) Animals like us. Verso, London

Roskies A (2002) Neuroethics for the New Millenium. Neuron 35:21-23

Russel W, Burch R (1959) The principles of humane experimental technique

Singer P (1975) animal liberation: towards an end to man's inhumanity to animals. Paladin Books, St Albans

Singer P (1995) Practical ethics, 2nd edn. Cambridge University Press, Cambridge

Stefansson H (2007) The biology of behaviour: scientific and ethical implications. EMBO reports vol 8 (Special Issue) 
Taylor P (1986) Respect for nature. Princeton University Press, Princeton

van der Staay FJ, Arndt SS, Nordquist RE (2009) Evaluation of animal models of neurobehavioral disorders. Behav Brain Functions 5:11

Warren MA (1997) moral status: obligations to persons and other living things. Clarendon Press, Oxford

Zutphen LFM, van Baumans V, Beynen AC (eds) (1993) Principles of laboratory animal science: a contribution to the humane use and care of animals and to the quality of experimental results. Elsevier, Amsterdam 